Bemt, L. van den, Luijks, H., Bor, H., Termeer, E., Lucassen, P., Schermer, T. Are asthma patients at increased risk of clinical depression? A longitudinal cohort study. Journal of Asthma: 2017, 53(1), 43-49

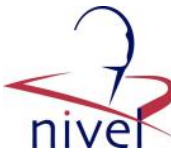

\begin{tabular}{|l|l}
$\begin{array}{l}\text { Postprint } \\
\text { Version }\end{array}$ & 1.0 \\
\hline Journal website & http://www.tandfonline.com/doi/full/10.3109/02770903.2015.1059852 \\
\hline Pubmed link & $\underline{\text { https://www.ncbi.nlm.nih.gov/pubmed/26313241 }}$ \\
\hline DOI & $10.3109 / 02770903.2015 .1059852$ \\
\hline
\end{tabular}

This is a NIVEL certified Post Print, more info at http://www.nivel.eu

\title{
Are asthma patients at increased risk of clinical depression? A longitudinal cohort study
}

\author{
LisetTe VAN DEN Bemt, PhD, HiLde LuiJKs, MD, HANs Bor, BSc, EVELIEN TERMEer, \\ MD, PETER LUCASSEN, PHD, MD, AND TJARD SCHERMER, PHD \\ Department of Primary and Community Care, Radboud University Medical Center, \\ Nijmegen, The Netherlands
}

\begin{abstract}
Objective: In this study, we assessed whether adult patients with asthma are more likely to be diagnosed with depression than diabetes patients or "healthy" controls during follow-up in primary care. Methods: Data from the Nijmegen Continuous Morbidity Registration were used to assess the risk for a first depression. Patients with asthma were compared with patients with diabetes and with two healthy controls matched on age, gender, socioeconomic status and attending general practice. With Cox proportional hazard analysis, we compared the risk of depression between these groups. These analyses were corrected for relevant covariates including a time-depending variable for multimorbidity. Explorative subgroup analyses were done for age, gender, socioeconomic status and multimorbidity. Results: Cumulative incidence of depression in asthma patients was $5.2 \%$, in DM patients $4.1 \%$ and in control subjects $3.3 \%$.

The hazard ratios for a first episode of depression in the asthma patients $\left(\mathrm{n}^{1 / 4795)}\right.$ compared to DM patients $\left(\mathrm{n}^{1 / 41033)}\right.$ and control subjects after correction for covariates were 1.11 (95\% CI 0.60-2.04) and 1.18 (95\% CI 0.781.79), respectively. Exploratory analyses showed that asthma patients without multimorbidity were at higher risk for a depression compared to reference groups, while asthma patients with multimorbidity were at lower risk for depression.

Conclusion: Asthma patients were not more likely to be diagnosed with a first depression compared to "healthy" control subjects or diabetes patients. The influence of multimorbidity on depression risk in asthma patients warrants further study.
\end{abstract}


Bemt, L. van den, Luijks, H., Bor, H., Termeer, E., Lucassen, P., Schermer, T. Are asthma patients at increased risk of clinical depression? A longitudinal cohort study. Journal of Asthma: 2017, 53(1), 43-49

\section{INTRODUCTION}

A co-existing depressive disorder has important implications for the management of patients with asthma, as asthma control, quality of life, dyspnea and life expectancy are lower in patients who suffer from depression [1-8]. On the other hand, asthma a chronic condition that can lead to dyspnea, impaired quality of life and feelings of helplessness during exacerbations - could trigger or augment depressive feelings [9$11]$.

High-depression prevalence rates in asthma patients as well as a strong association between depressive symptoms and asthma have been reported [2,7,12-17]. Solis and coworkers asked depressed asthma patients to report the age of onset of their asthma and their depression, and concluded that asthma preceded depression more often than vice versa (i.e. 62 versus 24\%) [18]. Other studies did not observe an association between asthma and depression $[10,19,20]$. The cross-sectional design of most studies does not shed light on the direction of this possible association $[10,12-$ $17,19,20]$.

A study by Walters et al. was the first to show that primary care asthma patients were more likely to be diagnosed with depression compared to matched non-asthmatic individuals, although the strength of the association was only moderate [2]. The presumed association between asthma and depression is subject to a number of caveats. Firstly, in most studies 'depression' merely represents a higher score on a depression questionnaire, which introduces the problem that symptoms of asthma and symptoms of depression can be entwined (for instance, fatigue can be the result of asthma, depression or both) [7,11-13]. Next, if asthma patients are indeed at higher risk of depression, the question remains if this is specific for asthma or a result of suffering from a chronic disease in general: increased depression rates have also been reported for other chronic diseases like ischemic heart pain, diabetes and epilepsy $[5,13,16,21]$. Finally, the depression risk could be different for relevant subgroups. For example, multimorbidity patterns differ between males and females [22].

The aim of the current paper is to assess whether adult patients with asthma are more likely to be diagnosed with adult-onset depression compared to patients with diabetes mellitus (DM) and "healthy" controls during continuous follow-up in primary care. We also looked at possible differences in this association between subgroups based on gender, socioeconomic status, age of asthma diagnosis and multimorbidity.

\section{METHODS}

\section{Study design}

The study was a dynamic historical cohort study using data from the Continuous Morbidity Registration (CMR) Nijmegen database. The CMR records all events of morbidity presented to the general practitioners (GPs) in four general practices in the Netherlands since 1971 [23,24]. Together the practices serve a population of approximately 13500 individuals with a low-turnover rate (55\% per year). The practice population is representative for the Dutch population in terms of age, gender and socioeconomic class [24]. Because all Dutch inhabitants are listed with a general practice and receive their medical care through this particular practice; general practice patient records provide a complete overview of the patient's medical history (including hospital care and diagnoses made by medical specialists in the hospital). 
Bemt, L. van den, Luijks, H., Bor, H., Termeer, E., Lucassen, P., Schermer, T. Are asthma patients at increased risk of clinical depression? A longitudinal cohort study. Journal of Asthma: 2017, 53(1), 43-49

Moreover, primary care is highly utilized in the Netherlands with a mean of five visits per patient per year. All the morbidities that are recorded in the CMR database are classified according to the UK Royal College of General Practitioners' E-book [25]. The GPs in the CMR practices use the criteria and definitions of the International Classification of Health Problems in Primary Care (ICHPPC-2) since 1985 to classify morbidity [26]. However, they continued to use the E-list categories to maintain consistent data collection.

Monthly meetings for all GPs involved are held to maintain the application of diagnostic criteria and discuss coding issues.

All enlisted patients of the CMR practices have been informed about the use of the database for research and could opt out. Studies based on CMR data comply with the Code of Conduct for Health Research, which has been approved by the Data Protection Authorities for conformity with the applicable Dutch privacy legislation. Since only de-identified information that is already available in the CMR records was used for the current study, approval of an external ethics committee was not required.

\section{Subject selection}

This study was based on all data of the CMR database from January 1985 (i.e. start of registration with ICHPPC-2 criteria) until December 2008. We included adult patients with asthma, DM and healthy controls. We excluded individuals that fulfilled one or more of the following criteria: (1) missing data (e.g. no socioeconomic status available), (2) unknown date of a depression diagnosis, (3) a first episode of depression before adulthood (before the age of 18), (4) less than 1month follow-up, (5) diagnosis of both asthma and DM and (6) severe other morbidity (i.e. congenital heart disease, congenital neurological diseases or Down's syndrome).

\section{Patients with asthma}

We considered all patients with asthma diagnosed since 1985 in the CMR database for this study (ICPC code R96). If asthma had been diagnosed before the age of 6, at least one subsequent asthma follow-up visit had to be recorded by the GP after the child's 6th birthday. Asthma patients were excluded if depression was diagnosed prior to or at the same time as their asthma was diagnosed. Observation time started when the asthma patient turned 18 or, in case of adult-onset asthma, at the time of diagnosis.

\section{Patients with diabetes}

We selected patients with DM type 1 or type 2 as a reference group suffering from another common chronic disease that is associated with a high-prevalence of depression [21].

Diabetes was selected because it has low mortality (510\% in 5 years), no rapid progression, no known intrinsic relation to depression and different symptoms than asthma. Again, observation time started when the patient turned 18 or at the time of diagnosis in case of adult-onset DM. DM patients with a diagnosis of depression prior to or at the same time of the DM diagnosis were excluded.

\section{Control subjects}


Bemt, L. van den, Luijks, H., Bor, H., Termeer, E., Lucassen, P., Schermer, T. Are asthma patients at increased risk of clinical depression? A longitudinal cohort study. Journal of Asthma: 2017, 53(1), 43-49

Patients with an asthma diagnosis were matched to control subjects drawn from the CMR cohort. Control subjects were free from any of the chronic conditions in Box 1 at the start of (but not necessarily later in) their observation period. These chronic conditions were selected because they affected at least 10000 Dutch residents and were severe enough to result in at least 10000 DALYs lost in 2011 [27]. We matched every asthma patient to two control subjects based on gender, date of birth ( \pm 2 years), general practice and socioeconomic status. The observation time of the matched controls started at the same point in time as the asthma patient's date of asthma diagnosis. If a first episode of depression in a potential control subject was recorded prior to or at the start date of the observation time of the asthma patient he or she was matched to, another control subject was selected. Obviously, control subjects could not develop asthma or DM during the observation period.

\section{Registration of depression in CMR database}

For this study, the date of a first episode of a physiciandiagnosed depression was considered an "event". It has been demonstrated that there is a high agreement $(85 \%)$ between a diagnosis of depression as recorded by the CMR GPs and the definition of major depressive disorder using the criteria in the Diagnostic and Statistical Manual of Mental Disorders, Fourth Edition (DSM-IV) [28]. Pre-adult onset depression (i.e. first episode of depression before the age of eighteen) was excluded from this study as it is different from adult onset depression in terms of severity, suicide risk, associated multimorbidity and treatment approach [29].

\section{[BOX 1]}

\section{Statistical analysis}

Characteristics of the asthma group were compared to the DM and control groups, and analyzed using _ 2 tests and unpaired Student's t tests. Incidence density rate for depression was the fraction of subjects per group that were diagnosed with a depression during the follow-up.

Cumulative uncorrected incident rate ratio (IRR) was calculated by comparing the cumulative incidence for the asthma group with the DM and control groups. The Cox proportional hazards analysis was used to identify the risk of an "event" (first depression) in the patients with asthma compared to the DM and control groups. The analyses with DM patients were corrected for age of start data collection, gender, socioeconomic status, attending general practice and multimorbidity. Multimorbidity was defined as the coexistence of one or more recorded chronic conditions (Box 1) and was entered as a dichotomous time-dependent covariate in the model (i.e. months of follow-up with multimorbidity compared to months of follow-up without multimorbidity).

The survival analysis comparing asthma patients with control subjects was corrected for multimorbidity during the followup only. The same analyses were done for relevant subgroups with at least 300 subjects. We considered gender, socioeconomic status, age of diagnosis (i.e. before the age of 18 versus 18 or older) and multimorbidity (yes/no) as subgroups.

Models were corrected for relevant covariates as described above except for the covariate that was used to divide the population in subgroups. Both uncorrected and corrected hazard ratio's (HRs) are presented. 
Bemt, L. van den, Luijks, H., Bor, H., Termeer, E., Lucassen, P., Schermer, T. Are asthma patients at increased risk of clinical depression? A longitudinal cohort study. Journal of Asthma: 2017, 53(1), 43-49

$\mathrm{p}$ Values used in all the analyses were two-tailed; p50.05 was considered statistically significant. Analyses were performed with SPSS version 18 (SPSS Inc., Chicago, IL).

\section{RESULTS}

The final dataset consisted of 795 patients with asthma, 1033 patients with diabetes and 1590 matched control subjects.

Figure 1 shows the subject selection for the study. Twentyfour subjects were excluded because of childhood-onset depression and of those subjects three had been diagnosed with asthma as well. None of the DM patients were excluded based on this criterion. We were unable to find two eligible control group matches for four asthma patients $(50.5 \%)$ and excluded these patients from the analyses.

The DM patients differed significantly from the asthma patients in terms of age, gender, socioeconomic status and attending practices (Table 1). Incidence density rate for a (physician diagnosed) first depression did not differ significantly between asthma and DM patients, but asthma patients did show a higher risk of depression compared to control subjects. The cumulative uncorrected IRR for depression in asthma patients compared to DM patients and to control subjects was 1.29 and 1.58 , respectively.

The HRs for a first episode of depression in the asthma patients compared to DM patients and control subjects after correction for covariates were 1.11 (95\% CI 0.602.04) and 1.18 (95\% CI 0.78-1.79), respectively (Table 2).

Tables 3 shows that the HR of depression in asthma patients without multimorbidity was 1.87 (95\% CI 1.16-3.02) compared to healthy controls, whereas the HR of depression in asthma patients with multimorbidity was 0.37 (95\% CI $0.16-0.86$ ) compared to control subjects. The same applied for the comparison with DM patients (Table 4). No other significant risk differences were found in the subgroup analyses.

\section{DISCUSSION}

The present study was performed to determine the risk of a first episode of physician-diagnosed depression in adult asthma patients compared to individuals who were either "healthy" at the start of their observation time or had another common chronic disease (i.e. diabetes). Based on our analyses, we cannot conclude that patients with asthma are at higher risk for depression. These findings do not support the general belief that asthma is a risk factor for depression.

Although we did not find a significantly higher risk of depression in asthma patients, our results are similar to the results of the only other comparable study [2]. In this particular study, the incident rate ratio (IRR) was calculated and the uncorrected IRR for a physician-diagnosed depression was 1.63 for asthma compared to healthy controls, which is very similar to our findings (i.e. 1.58).

A limitation is that we had no information available on asthma disease severity, level of asthma control, smoking rate and obesity. They may, however, be potential confounders.

Patients with frequent exacerbations, moderate-to-severe asthma or uncontrolled asthma may be at higher risk of depression than patients with stable, intermittent-tomild or controlled asthma. This warrants further exploration. We also did not have information on obesity and smoking rate, which are both associated with higher asthma severity as well as with depression and could therefore be important 
Bemt, L. van den, Luijks, H., Bor, H., Termeer, E., Lucassen, P., Schermer, T. Are asthma patients at increased risk of clinical depression? A longitudinal cohort study. Journal of Asthma: 2017, 53(1), 43-49

confounders [30,31]. Careful registration of these lifestyle characteristics has increased in the last decades, but as the data collection in the CMR already started in the 1980s, we could not correct for these factors.

We did, however, correct for the time that patients suffered from comorbidity during their observation time. Inclusion of a time-depending covariate is an important advantage of the chosen statistical technique and makes optimal use of our longitudinal valid primary care data for more than 30 years.

However, even the use of this covariate does not fully encompass the complexity of multimorbidity, we included only a selection of comorbid conditions [27]. Also, severity of these conditions is not recorded in the CMR and could influence depression risk as well $[16,32]$. Next, the risk of depression increases with the number of chronic conditions [32]. Considering the sharp increase in the number of patients with multimorbidity and the results of our subgroup analysis, we would encourage further research on the effect of multimorbidity on depression risk in asthma.

We used depression as diagnosed by the GP as the outcome for our study. The prevalence of depression is considered to be underestimated as not all depressed patients present their problem to a GP and GPs do not always diagnose depression when presented [33]. However, a depression diagnosed by a GP reflects a relevant depression because the patient has a reason for encounter. Then again, usually participating patients are asked to fill out a questionnaire on depressive

\section{[FIGURE 1] [TABLE 1] [TABLE 2] [TABLE 3] [TABLE 4]}

symptoms which could result in an overestimation of depression risk [7,11-13]. The advantage of our definition is that the diagnosis is clinically relevant as the patient considered it necessary to present the problem to the GP. This is reflected by the fact that $30-50 \%$ of depression cases that were "'missed" by GPs had the lowest possible "depression score" on a depression questionnaire and must be considered as very mild or even "doubtful" depression cases [34]. We used a large primary care cohort with extensive follow-up, with almost 800 patients with asthma and matching numbers of controls. Since all residents in the Netherlands are registered in a general practice, prevalence and incidence figures based on primary care registrations are representative for the general population. While most previous studies have only looked at the cross-sectional association between asthma and depression $[10,12-$ $17,19,20]$, in our current study we were able to study the temporal relation between asthma and depression.

Based on our study, we cannot conclude that patients with asthma are at higher risk for depression. Nonetheless, there is little doubt about the implications of depression on morbidity in patients with asthma [1-8]. There are some studies showing positive effects of "depression" management (for instance, with pharmacotherapy or aerobic training) on asthma morbidity and psychosocial morbidity $[35,36]$.

\section{CONCLUSION}

Asthma patients were not more likely to have a first diagnosis of depression during the follow-up compared to these reference groups. More research is needed to better understand the ambiguous role of multimorbidity in depression risk of asthma patients. 
Bemt, L. van den, Luijks, H., Bor, H., Termeer, E., Lucassen, P., Schermer, T. Are asthma patients at increased risk of clinical depression? A longitudinal cohort study. Journal of Asthma: 2017, 53(1), 43-49

\section{Acknowledgements}

We would like to thank all GPs that registered for the Continuous Morbidity

Registration in the past 40 years.

Moreover, we would like to thank Joep van der Meer for his contribution to the initial analysis of the data. Finally, we would like to thank Reinier Akkermans for his assistance in the statistical analysis.

\section{Declaration of interest}

The authors did not receive funding for the reported study. All authors certify that there is no conflict of interest regarding the submitted manuscript.

\section{REFERENCES}

1. Oga T, Nishimura K, Tsukino M, Sato S, Hajiro T, Mishima M. Analysis of longitudinal changes in the psychological status of patients with asthma. Respir Med 2007;101:21332138.

2. Walters $P$, Schofield $P$, Howard L, Ashworth M, Tylee A. The relationship between asthma and depression in primary care patients: a historical cohort and nested case control study. PLoS One 2011;6:e20750.

3. Lavoie KL, Cartier A, Labrecque M, Bacon SL, Lemiere C, Malo JL, Lacoste G, et al. Are psychiatric disorders associated with worse asthma control and quality of life in asthma patients? Respir Med 2005;99:1249-1257.

4. Lavoie KL, Bacon SL, Barone S, Cartier A, Ditto B, Labrecque M. What is worse for asthma control and quality of life: depressive disorders, anxiety disorders, or both? Chest 2006;130:1039-1047.

5. Moussavi S, Chatterji S, Verdes E, Tandon A, Patel V, Ustun B. Depression, chronic diseases, and decrements in health: results from the World Health Surveys. Lancet 2007;370:851-858.

6. Di Marco F, Verga M, Santus P, Giovannelli F, Busatto P, Neri M, Girbino G, et al. Close correlation between anxiety, depression, and asthma control. Respir Med 2010;104:22-28.

7. Eisner MD, Katz PP, Lactao G, Iribarren C. Impact of depressive symptoms on adult asthma outcomes. Ann Allergy Asthma Immunol 2005;94:566-574.

8. Neuman A, Gunnbjornsdottir M, Tunsater A, Nystrom L, Franklin KA, Norrman E, Janson C. Dyspnea in relation to symptoms of anxiety and depression: a prospective population study. Respir Med 2006;100:1843-1849.

9. Goldney RD, Ruffin R, Fisher LJ, Wilson DH. Asthma symptoms associated with depression and lower quality of life: a population survey. Med J Aust 2003;178:437-441.

10. Janson C, Bjornsson E, Hetta J, Boman G. Anxiety and depression in relation to respiratory symptoms and asthma. Am J Respir Crit Care Med 1994;149:930-934.

11. Katz PP, Morris A, Julian L, Omachi T, Yelin EH, Eisner MD, Blanc PD. Onset of depressive symptoms among adults with asthma: results from a longitudinal observational cohort. Prim Care Respir J 2010;19:223-230.

12. Carvalho NS, Ribeiro PR, Ribeiro M, Nunes MP, Cukier A, Stelmach R. Comparing asthma and chronic obstructive pulmonary disease in terms of symptoms of anxiety and depression. J Bras Pneumol 2007;33:1-6.

13. Filipcic I, Popovic-Grle S, Marcinko D, Basic S, Hotujac L, Pavicic F, Hajnaek S, Aganovic' I. Screening for depression disorders in patients with chronic somatic illness. Coll Antropol 2007;31:139-143.

14. Goodwin RD, Jacobi F, Thefeld W. Mental disorders and asthma in the community. Arch Gen Psychiatry 2003;60:1125-1130.

15. Scott KM, Von KM, Ormel J, Zhang MY, Bruffaerts R, Alonso J, Kessler RC, et al. Mental disorders among adults with asthma: results from the World Mental Health Survey. Gen Hosp Psychiatry 2007;29:123-133.

16. Farmer A, Korszun A, Owen MJ, Craddock N, Jones L, Jones I, Gray J, et al. Medical disorders in people with recurrent depression. Br J Psychiatry 2008;192:351-355.

17. Nascimento I, Nardi AE, Valenca AM, Lopes FL, Mezzasalma MA, Nascentes R, Zin WA. Psychiatric disorders in asthmatic outpatients. Psychiatry Res 2002;110:73-80. 
Bemt, L. van den, Luijks, H., Bor, H., Termeer, E., Lucassen, P., Schermer, T. Are asthma patients at increased risk of clinical depression? A longitudinal cohort study. Journal of Asthma: 2017, 53(1), 43-49

18. Solis OL, Khan DA, Brown ES. Age at onset of major depression in inner-city adults with asthma. Psychosomatics 2006;47:330-332.

19. Goodwin RD, Olfson M, Shea S, Lantigua RA, Carrasquilo O, Gameroff MJ, Weissman MM. Asthma and mental disorders in primary care. Gen Hosp Psychiatry 2003;25:479_ 483.

20. Wainwright NW, Surtees PG, Wareham NJ, Harrison BD. Psychosocial factors and asthma in a community sample of older adults. J Psychosom Res 2007;62:357-361.

21. Anderson RJ, Freedland KE, Clouse RE, Lustman PJ. The prevalence of comorbid depression in adults with diabetes: a meta-analysis. Diabetes Care 2001;24:1069-1078.

22. Schafer I, Hansen H, Schon G, Hofels S, Altiner A, Dahlhaus A, Gensichen J, et al. The influence of age, gender and socioeconomic status on multimorbidity patterns in primary care. First results from the multicare cohort study. BMC Health Serv Res 2012;12:89.

23. van Weel C, de Grauw W. Family practices registration networks contributed to primary care research. J Clin Epidemiol 2006;59: 779-783.

24. Van Weel C. The Continuous Morbidity Registration Nijmegen: background and history of a Dutch general practice database. Eur J Gen Pract 2008;14:5-12.

25. Hodgkin K. Towards earlier diagnosis in primary care. Edinburgh, London, and New York: Churchill Livingstone; 1966.

26. Classification Committee of World Organisation of NationalColleges A, Academic Associations of GeneralPractitioners/Family P. ICHPPC-2-Defined. Inclusion criteria for the use of the rubrics of the International Classification of Health Problems in Primary Care. Oxford: Oxford University Press; 1983.

27. Nationaal Kompas Volksgezondheid, versie 4.6.1 Utrecht: RIVM; 2012 [updated 31-012012; cited 2012 07-02-2012]. Available from: www.nationaalkompas.nl.

28. Weel-Baumgarten EM, van den Bosch WJ, van den Hoogen HJ, Zitman FG. The validity of the diagnosis of depression in general practice: is using criteria for diagnosis as a routine the answer? Br J Gen Pract 2000;50:284-287.

29. Korczak DJ, Goldstein BI. Childhood onset major depressive disorder: course of illness and psychiatric comorbidity in a community sample. J Pediatr 2009;155:118-123.

30. Goodwin RD. Intergenerational transmission of chronic physical disease via chronic mental disorders: the potential role of addictive behaviors. Addict Behav 2008;33:14321440.

31. Hasler G, Gergen PJ, Ajdacic V, Gamma A, Eich D, Rossler W, Angst J. Asthma and body weight change: a 20 -year prospective community study of young adults. Int J Obes (Lond) 2006; 30:1111-1118.

32. Gunn JM, Ayton DR, Densley K, Pallant JF, Chondros P, Herrman HE, Dowrick CF. The association between chronic illness, multimorbidity and depressive symptoms in an Australian primary care cohort. Soc Psychiatry Psychiatr Epidemiol 2012;47: 175-184.

33. Piek E, Nolen WA, van der Meer K, Joling KJ, Kollen BJ, Penninx BW, van Marwijk HW, van Hout HP. Determinants of (non-) recognition of depression by general practitioners: results of the Netherlands Study of Depression and Anxiety. J Affect Disord 2012;138:397404.

34. Thompson C, Ostler K, Peveler RC, Baker N, Kinmonth AL. Dimensional perspective on the recognition of depressive symptoms in primary care: the Hampshire Depression Project 3. Br J Psychiatry 2001;179:317-323.

35. Brown ES, Howard C, Khan DA, Carmody TJ. Escitalopram for severe asthma and major depressive disorder: a randomized, double-blind, placebo-controlled proof-of-concept study. Psychosomatics 2012;53:75-80.

36. Mendes FA, Goncalves RC, Nunes MP, Saraiva-Romanholo BM, Cukier A, Stelmach R, Jacob-Filho W, et al. Effects of aerobic training on psychosocial morbidity and symptoms in patients with asthma: a randomized clinical trial. Chest 2010;138:331-337. 
Bemt, L. van den, Luijks, H., Bor, H., Termeer, E., Lucassen, P., Schermer, T. Are asthma patients at increased risk of clinical depression? A longitudinal cohort study. Journal of Asthma: 2017, 53(1), 43-49

BOX, TABLES AND FIGURE

Box 1. Chronic conditions.

Asthma, diabetes, malignant neoplasms, chronic obstructive pulmonary disease (COPD), ulcerative colitis, Crohn's disease, ischemic heart disease, congestive heart failure, stroke, arthritis (osteoarthritis, rheumatoid arthritis, ankylosing spondylitis and polyarthritis), osteoporosis, multiple sclerosis, Parkinson's disease, anxiety disorders, dementia, epilepsy, hearing disorder, and/or atopic eczema 


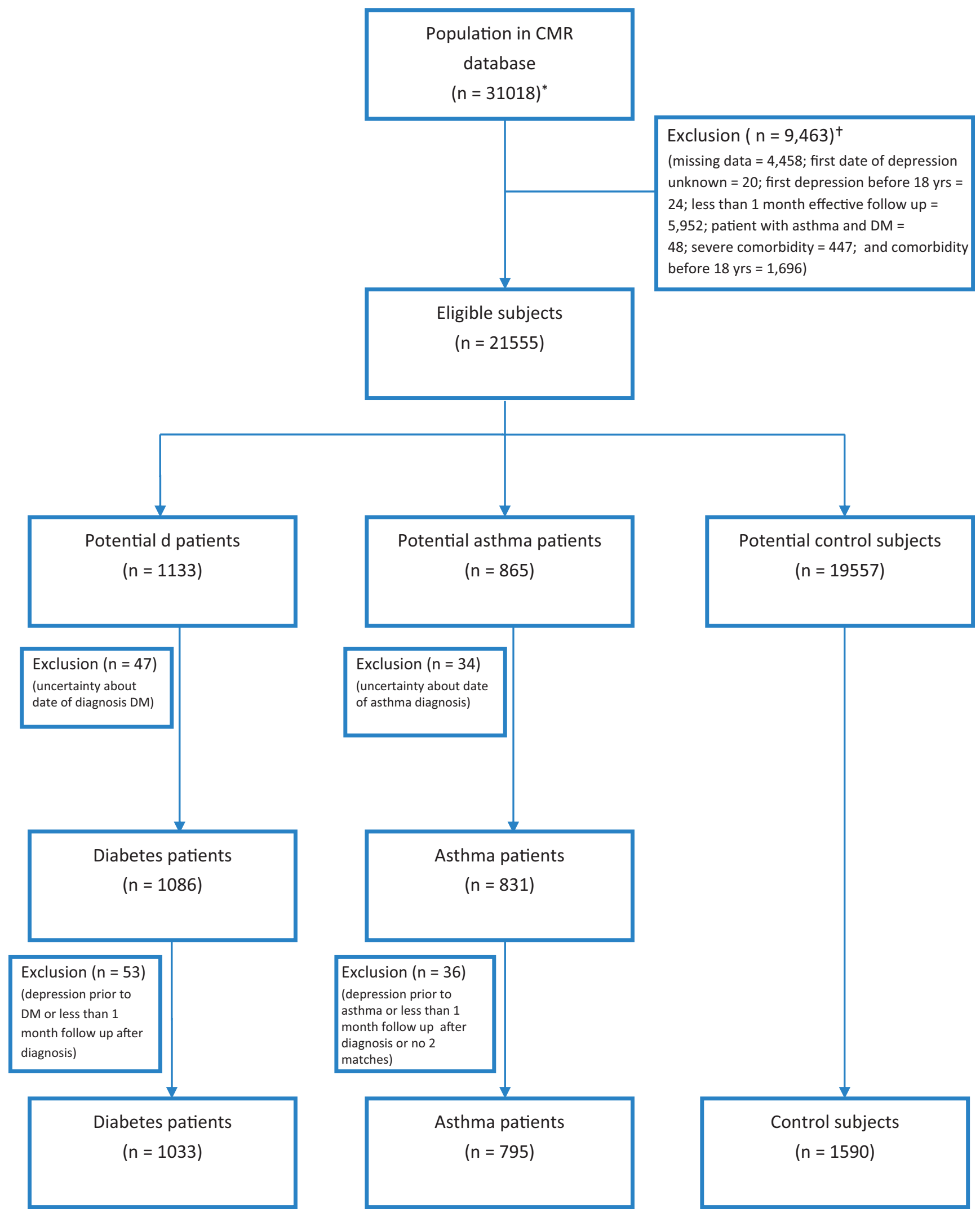

Figure 1. Flow chart of selection procedure of asthma patients, diabetes patients and control subjects in Continuous Morbidity Registration (CMR) Nijmegen Database. *Total number of patients registered at one of the participating CMR practices between 1985 and 2008 at any given time. $†$ Several subjects with more than one exclusion criterion. 
Bemt, L. van den, Luijks, H., Bor, H., Termeer, E., Lucassen, P., Schermer, T. Are asthma patients at increased risk of clinical depression? A longitudinal cohort study. Journal of Asthma: 2017, 53(1), 43-49

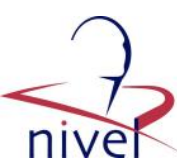

Table 1. Characteristics of patients with asthma, patients with DM and control subjects with no chronic conditions at the start of follow-up.

\begin{tabular}{lccc}
\hline & Asthma $(n=795)$ & DM $(n=1033)$ & Controls $(n=1590)$ \\
\hline Cumulative incidence of depression & $41(5.2 \%)$ & $42(4.1 \%)$ & $53(3.3 \%)^{*}$ \\
Gender & $324(41.1 \%)$ & $530(51.3 \%)^{* *}$ & $654(41.1 \%)$ \\
$\quad$ Male & $33.2(13.3)$ & $62.2(14.7)^{* *}$ & $33.4(13.1)$ \\
$\quad$ Age start of follow up, years & $279(35.1 \%)$ & $758(73.4 \%)^{* *}$ & $242(15.2 \%)^{* *}$ \\
Occurrence of comorbid conditions & & & \\
$\quad$ during follow up & & & \\
Socioeconomic status & $275(34.6 \%)$ & $529(51.2 \%)^{* *}$ & $550(34.6 \%)$ \\
$\quad$ Low & $359(45.2 \%)$ & $389(37.7 \%)$ & $718(45.2 \%)$ \\
Middle & $161(20.3 \%)$ & $115(11.1 \%)$ & $322(20.3 \%)$ \\
$\quad$ High & $234(29.4 \%)$ & $300(29.0 \%)^{*}$ & \\
General practice & $160(20.1 \%)$ & $222(21.5 \%)$ & $468(29.4 \%)$ \\
$\quad 1$ & $130(16.4 \%)$ & $211(20.4 \%)$ & $320(20.1 \%)$ \\
2 & $271(34.1 \%)$ & $300(29.0 \%)$ & $260(16.4 \%)$ \\
3 & & & $542(34.1 \%)$ \\
4 & &
\end{tabular}

Data are presented as number (\%) or mean (SD).

$* p<0.05$ for difference with asthma group.

$* * p<0.01$ for difference with asthma group.

aComorbid conditions: malignant neoplasms, chronic obstructive pulmonary disease (COPD), ulcerative colitis, Crohn's disease, ischemic heart disease, congestive heart failure, stroke, arthritis (osteoarthritis, rheumatoid arthritis, ankylosing spondylitis and polyarthritis), osteoporosis, multiple sclerosis, Parkinson's disease, anxiety disorders, dementia, epilepsy, hearing disorder, and/or atopic eczema.

Table 2. Cox' proportional HRs for risk of first depression in asthma patients compared to diabetes patients and controls.

\begin{tabular}{lcc}
\hline & Uncorrected HR & Corrected HR \\
\hline Asthma versus diabetes & $1.22(0.79-1.87)$ & $1.11(0.60-2.04)^{\mathrm{a}}$ \\
Asthma versus control subjects & $1.27(0.75-1.70)$ & $1.18(0.78-1.79)^{\mathrm{b}}$ \\
\hline
\end{tabular}

Results are shown as HR (95\% CI).

${ }^{a}$ Corrected for gender, general practice, social economic status, age and months of multimorbidity.

${ }^{b}$ Corrected for months of multimorbidity. 
Bemt, L. van den, Luijks, H., Bor, H., Termeer, E., Lucassen, P., Schermer, T. Are asthma patients at increased risk of clinical depression? A longitudinal cohort study. Journal of Asthma: 2017, 53(1), 43-49

Table 3. Cokx' proportional HRs for risk of first depression in asthma patients compared to diabetes patients for subgroups.

\begin{tabular}{lrrr}
\hline & $N$ & Uncorrected HR & Corrected HR \\
\hline Gender & & & \\
$\quad$ Males & 785 & $0.71(0.29-1.77)$ & $0.39(0.11-1.43)$ \\
$\quad$ Females & 969 & $1.36(0.83-2.25)$ & $1.48(0.73-2.99)$ \\
Age (dummy date of) diagnosis & & \\
$\quad$ Before 18 years old & $134^{\mathrm{b}}$ & & \\
$\quad \begin{array}{l}\mathrm{a} \text { 18 years old } \\
\text { Socio-economic status }\end{array}$ & 1684 & $1.18(0.75-1.86)$ & $1.05(0.56-1.98)$ \\
$\quad$ & & & \\
$\quad$ Low & 803 & $1.30(0.72-2.33)$ & $0.95(0.40-2.25)$ \\
$\quad$ Middle & 746 & $0.93(0.46-1.91)$ & $0.78(0.30-2.04)$ \\
$\quad$ High & $263^{\mathrm{b}}$ & & \\
Multimorbidity & & & \\
$\quad$ Yes & 1026 & $0.51(0.23-1.13)$ & $0.39(0.14-1.10)$ \\
$\quad$ No & 788 & $1.40(0.73-2.72)$ & $2.45^{*}(1.00-5.98)$ \\
\hline
\end{tabular}

Results are shown as HR (95\% CI).

${ }^{a}$ Corrected for gender, general practice, social economic status, age, and months of multimorbidity if applicable (i.e. no correction for the subgroup variable).

${ }^{\mathrm{b}}$ No analysis due to small group size $(n<300)$.

$* p<0.05$. 
Bemt, L. van den, Luijks, H., Bor, H., Termeer, E., Lucassen, P., Schermer, T. Are asthma patients at increased risk of clinical depression? A longitudinal cohort study. Journal of Asthma: 2017, 53(1), 43-49

Table 4. Cox' proportional HRs for risk of first depression in asthma patients compared to controls for subgroups.

\begin{tabular}{lrlc}
\hline & $N$ & Uncorrected HR & Corrected HR ${ }^{\mathrm{a}}$ \\
\hline Gender & & & \\
$\quad$ Males & 958 & $0.74(0.30-1.82)$ & $0.83(0.34-2.05)$ \\
$\quad$ Females & 1383 & $1.30(0.81-2.07)$ & $1.36(0.85-2.18)$ \\
Age (dummy date of) diagnosis & & \\
$\quad$ Before 18 years old & $228^{\mathrm{b}}$ & & \\
$\quad$ 18 years old & 2107 & $1.02(0.66-1.57)$ & $1.07(0.69-1.67)$ \\
Socio-economic status & & & \\
$\quad$ Low & 800 & $1.34(0.72-2.51)$ & $1.35(0.72-2.56)$ \\
$\quad$ Middle & 1060 & $0.81(0.42-1.56)$ & $0.91(0.46-1.77)$ \\
$\quad$ High & 469 & $1.63(0.59-4.52)$ & $1.65(0.59-4.62)$ \\
Multimorbidity & & & \\
$\quad$ Yes & 520 & $0.37 *(0.16-0.85)$ & $0.37 *(0.16-0.86)$ \\
$\quad$ No & 1830 & $1.87^{*}(1.16-3.02)$ & NA \\
\hline
\end{tabular}

Results are shown as HR (95\% CI).

aCorrected for months of multimorbidity if applicable (i.e. no correction for the subgroup "no multimorbidity").

bo analysis due to small group size $(n<300)$.

$* p<0.05$. 